\title{
Effects of scattering parameters on charge-sign dependent cosmic ray modulation
}

\author{
M.D. Ngobeni ${ }^{1}$ \\ School of Physical and Chemical Sciences \\ North-West University, 2745 Mmabatho, South Africa \\ Centre for Space Research \\ North-West University, 2520 Potchefstroom, South Africa \\ E-mail: Donald.Ngobenienwu.ac.za
}

\section{M.S. Potgieter}

Centre for Space Research

North-West University, 2520 Potchefstroom, South Africa

E-mail: Marius.Potgieter@nwu.ac.za

\section{O.P.M. Aslam}

Centre for Space Research

North-West University, 2520 Potchefstroom, South Africa

E-mail: as lamk1r2003@gmail.com

\section{Bisschoff}

Centre for Space Research

North-West University, 2520 Potchefstroom, South Africa

E-mail: driaanbegmail.com

\begin{abstract}
A comprehensive three-dimensional (3D) numerical drift model is used to compute the modulation of cosmic ray protons and anti-protons in the heliosphere. This is done using the latest development in deriving diffusion coefficients and cosmic ray interstellar spectra. Emphasis is placed on the effects of the spatial dependence of the scattering parameter, $\omega \tau$, with $\omega$ the gyro-frequency and $\tau$ a time scale defined by diffusive scattering, on the drift coefficient in the modulation of the mentioned species. A comparison of the numerical modelling of the ratio of anti-protons to protons obtained from different scenarios of $\omega \tau$ is made over a solar cycle. It is found that effects of the spatial dependence of $\omega \tau$ on the drift scale in the subsequent anti-proton to proton ratio are more prominent in the $A>0$ solar magnetic polarity cycle. This charge-sign dependent modulation study should assist in establishing the amount of drifts observed at Earth from minimum to maximum solar activity during both polarity cycles.
\end{abstract}

36th International Cosmic Ray Conference -ICRC2019-

July 24th - August 1st, 2019

Madison, WI, U.S.A.

\footnotetext{
${ }^{1}$ Speaker

(C) Copyright owned by the author(s) under the terms of the Creative Commons

Attribution-NonCommercial-NoDerivatives 4.0 International License (CC BY-NC-ND 4.0).
} 


\section{Introduction}

An impressive amount of PAMELA and AMS measurements of the ratio of anti-protons to protons, as oppositely charged galactic cosmic rays (GCRs), are now available with increased accuracy down to $\sim 0.5 \mathrm{GeV}[1 ; 2]$. This ratio is of crucial importance for understanding: (1) the propagation of GCRs with a different charge-sign in the heliosphere, and (2) the fundamental physical processes occurring in our Galaxy. For the latter, the established paradigm of antiproton production is that physical processes in our Galaxy combined with the interstellar spectrum for protons determine the anti-proton local interstellar spectrum (LIS). This is mainly due to the fact that the process producing anti-protons is the collisions of high energy cosmic ray protons with the interstellar medium - an active field of research on its own with exciting developments, See discussions by $[3 ; 4 ; 17 ; 18 ; 19]$.

When inside the heliosphere GCRs encounter an outward moving and expanding solar wind with cyclic magnetic field fluctuations and turbulence. The understanding of this interaction is currently based on four major modulation processes: convection, diffusion, adiabatic energy changes, and gradient, curvature and current sheet drifts. Combined, these interplaying processes cause the intensity of GCRs to decrease toward the Sun and to change significantly over its 11-year solar activity cycle. See the review by [5] for a concise discussion. Because protons and anti-protons access the inner heliosphere from different regions because of particle drifts, their intensities evolve differently over a 22-year magnetic polarity cycle.

From a numerical modelling point of view, the global modulation of anti-protons and protons has been addressed, although at varying levels of complexity (e.g. [2; 6; 7; 19]). From these studies it has been established that the anti-proton to proton ratio strongly depends on energy (rigidity) as well as on solar activity. An aspect that has been overlooked is separating the subsequent charge-sign dependent modulation effects on the drift coefficient of the spatial dependence of the scattering parameters, $\omega \tau$, from its rigidity dependence, with $\omega$ the gyrofrequency of the particles and $\tau$ a time scale defined by diffusive scattering. This remains so because establishing the rigidity and spatial dependence of $\omega \tau$ properly requires an elaborate turbulence theory throughout the entire heliosphere; which is not yet possible. However, progress is being made in this regard (e.g. $[8 ; 9 ; 10 ; 11])$.

In this paper we present a theoretical study of the subsequent charge-sign modulation effects of the spatial dependence of the scattering parameters $(\omega \tau)$, excluding contribution by its rigidity dependence, on the drift coefficient. As a first step, this study is done on the modulation of anti-protons and protons over an extended energy range for both solar magnetic polarity cycles and as solar activity increases from minimum to moderate maximum modulation conditions. The numerical modelling is done with a three-dimensional (3D) drift-model described by [12]. The proton and anti-proton LISs are based on a Galactic Propagation Model [13]; with the proton LIS constrained by Voyager 1 observations at lower kinetic energies (< $\sim 200 \mathrm{MeV}$ ) and PAMELA observations at higher energies similar to [12] and [16].

\section{The heliospheric drift coefficient}

In general, the average drift velocity caused by the gradients and curvature in the global heliospheric magnetic field (HMF) is given by 


$$
\begin{aligned}
\left\langle\mathbf{v}_{d}\right\rangle & =\nabla \times K_{T} \frac{\boldsymbol{B}}{B_{m}} \\
& =\frac{\beta P}{3}\left[f_{s} \nabla \times \frac{\boldsymbol{B}}{B_{m}^{2}}+\nabla f_{s} \times \frac{\boldsymbol{B}}{B_{m}^{2}}\right],
\end{aligned}
$$

with $K_{T}$ the generalized drift coefficient (in some reports indicated as $K_{A}$ ); $\boldsymbol{B}$ is the HMF vector with magnitude $B_{m}, f_{s}$ is the drift reduction factor due to diffusive scattering, $P$ is the GCR particle's rigidity and $\beta$ is the ratio of this particle's speed to the speed of light; $B_{m}$ is usually assumed to be a modified Parker HMF $[5 ; 12 ; 16]$.

Under the assumption that $f_{\mathrm{s}}$ has no spatial dependence, the average drift velocity in Eq. (1) can be written as:

$$
\left\langle\mathbf{v}_{d}\right\rangle=\nabla \times f_{s} \frac{\beta P}{3 B_{m}} \frac{\boldsymbol{B}}{B_{m}} .
$$

The components of interest of the average drift velocity as they appear in Eq. (2) are:

$$
\left\langle\mathbf{v}_{d}\right\rangle_{r}=-\frac{1}{r \sin \theta} \frac{\partial}{\partial \theta}\left(\sin \theta K_{T} \sin \psi\right) \boldsymbol{e}_{r} \text { and }\left\langle\mathbf{v}_{d}\right\rangle_{\theta}=-\frac{1}{r} \frac{\partial}{\partial r}\left(r K_{T} \sin \psi\right) \boldsymbol{e}_{\theta},
$$

with $\psi$ the spiral angle and $K_{T}$ given as

$$
K_{T}=f_{s} \frac{\beta P}{3 B_{m}} \text {. }
$$

However, when $\nabla f_{s} \neq 0$, these components become

$$
\begin{aligned}
\left\langle\mathbf{v}_{d}\right\rangle_{r} & =[-\frac{1}{r \sin \theta} \frac{\partial}{\partial \theta}\left(\sin \theta K_{T} \sin \psi\right)-\overbrace{\frac{1}{r} \frac{\partial f_{s}}{\partial \theta} \frac{\beta P}{3 B_{m}} \sin \psi}^{\text {New term }}] \boldsymbol{e}_{r} \\
\left\langle\mathbf{v}_{d}\right\rangle_{\theta} & =[-\frac{1}{r} \frac{\partial}{\partial r}\left(r K_{T} \sin \psi\right)+\overbrace{\frac{\partial f_{s}}{\partial r} \frac{\beta P}{3 B_{m}} \sin \psi}^{\text {New term }}] \boldsymbol{e}_{\theta} .
\end{aligned}
$$

In what follows, the spatial dependence alone of $\omega \tau$ is constructed from what is currently known to study the effects of the new terms in Eq. (5). In this way the rigidity dependence of $\omega \tau$ is neglected.

\section{Spatial dependence of $\omega \tau$ and $f_{s}$}

A functional form of $f_{s}$ that only contains spatial dependence can be easily constructed from the numerical Monte Carlo test particle simulation code of [9]. In their simulation they calculated $K_{T}$ by integrating the trajectories of charged particles that are scattered in turbulent magnetic fields. The best fit to their simulated $K_{T}$ is given by the following expression:

$$
K_{T}=\frac{\beta P}{3 B_{m}} \frac{1}{1+a\left(\frac{\delta B^{2}}{B_{m}^{2}}\right)^{b}} .
$$

Here, $\delta B^{2}$ is the magnetic variance. For a composite model with $15 \%$ slab and $85 \% 2 \mathrm{D}$ they achieved good correlation between numerical simulations and Eq. (6) when $a=1.09 \pm 0.52$ and $b=0.81 \pm 0.35$. Following Eq. (4), the corresponding $f_{s}$ can be written as 


$$
f_{s}=\frac{1}{1+a\left(\frac{\delta B^{2}}{B_{m}^{2}}\right)^{b}} .
$$

It thus become possible to relate $f_{s}$ in Eq.(7) to $\omega \tau$. Following [14], the general expression for $f_{s}$ is given as

$$
f_{s}=\frac{(\omega \tau)^{2}}{1+(\omega \tau)^{2}} .
$$

Therefore, the corresponding $\omega \tau$ can be approximated as

$$
\omega \tau=\frac{1}{\sqrt{a}\left[\frac{\delta B^{2}}{B_{m}^{2}}\right]^{\frac{b}{2}}} .
$$

Note that the drift reduction in this study, as given by Eq. (7), does not alter the power law rigidity dependence of the weak scattering drift coefficient below $1.0 \mathrm{GV}$; a serious requirement for reproducing observations of GCRs over a wide range of energy and from solar minimum to moderate solar maximum conditions (see $[12 ; 16])$. However, it remains to be seen what effects on GCR modulation the spatial depence of $\omega \tau$ and $f_{s}$, as given by Eqs. (7) and (9), will produce which is the main purpose of this study.
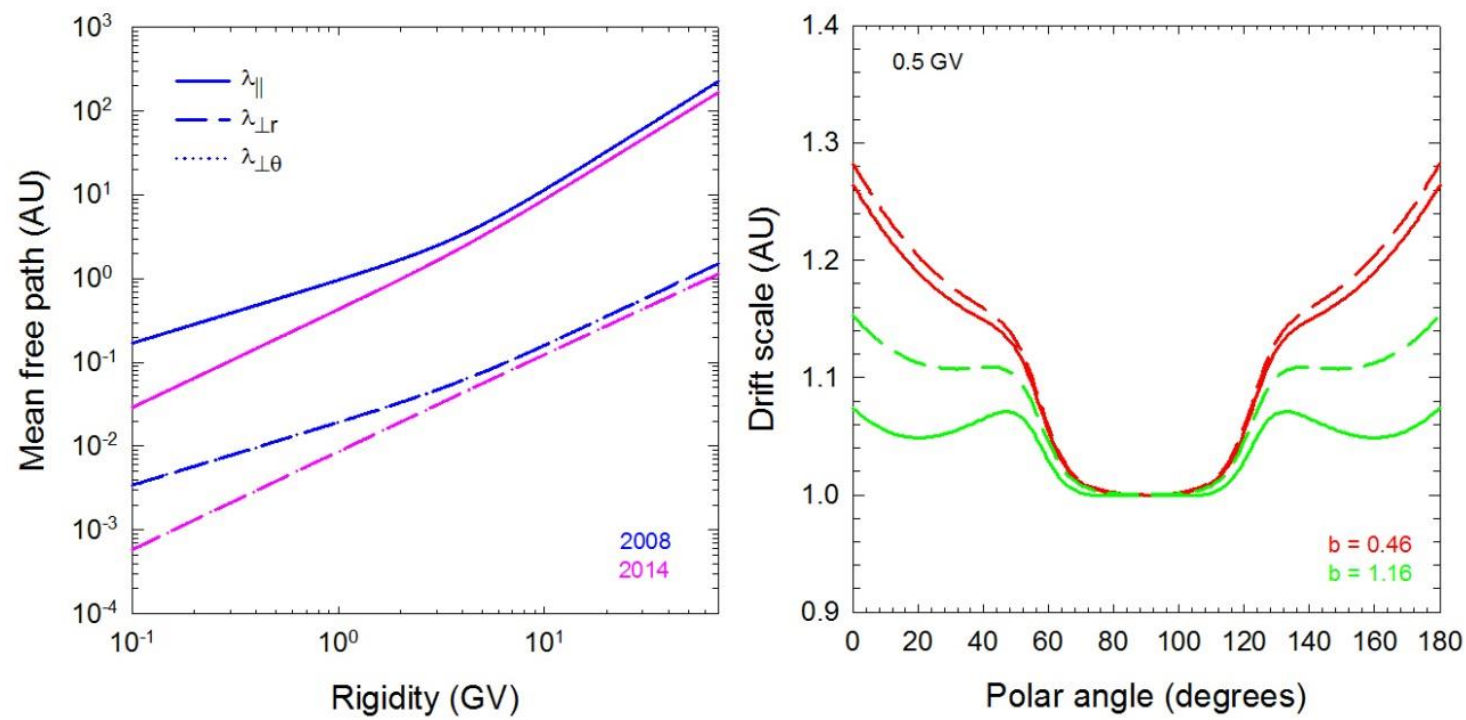

Fig.1: Left panel: Mean free paths (MFPs in AU) are shown as required for the observed proton and antiproton modulation during 2008 (blue lines) and 2014 (pink lines), as a function of rigidity at the Earth ( radial distance of $1 \mathrm{AU}$ in the equatorial plane). Solid, dashed and dotted lines represent $\left(\lambda_{\|}\right)$, the parallel MFP and the perpendicular MFPs in the radial $\left(\lambda_{\perp r}\right)$ and polar $\left(\lambda_{\perp \theta}\right)$ directions, respectively. Right panel: Normalized drift scale ( $\lambda_{A}$ in AU), corresponding to the two scenarios of $f_{s}$ in Eq. (9); $b=0.46$ for red lines and $b=1.16$ for green lines, as a function of polar angle for $0.5 \mathrm{GV}$ at $1 \mathrm{AU}$. The solid lines and dashed lines represent the assumed drift scale in 2008 and 2014, respectively.

The left panel of Fig. (1) shows the rigidity dependence of the mean free paths (MFPs) used in this study for the modulation conditions of 2008 (blue lines) and 2014 (pink lines) as done below. Parallel MFP $\left(\lambda_{\|}\right)$is represented by solid lines, while the perpendicular MFPs in the radial $\left(\lambda_{\perp r}\right)$ and polar directions $\left(\lambda_{\perp \theta}\right)$ are represented by long dashed and dotted lines, respectively. The rigidity dependence of these MFPs, as depicted in Fig. (1), is required to reproduce PAMELA proton observations from 2006 to 2009 ([12]) and 2014 ([16]). The right 
panel of Fig. (1) shows two scenarios of the drift scale, $\lambda_{A}$, normalized to their values in the equatorial plane, as a function of polar angle for a rigidity of $0.5 \mathrm{GV}$ at $1 \mathrm{AU}$. The solid and dashed lines represent the assumed values of $\lambda_{A}$ corresponding to 2008 and 2014, respectively. Here, the red lines and green lines respectively denote the computed and normalized $\lambda_{A}$ as studied in this work, obtained with $b=0.46$ and $b=1.16$ in Eq. (9). For both scenarios $a=0.57$ and $\delta B^{2}=B_{m}\left(25+25 \cos ^{2} \theta\right) r^{-2.7}$ is assumed. The normalization of $\lambda_{A}$ is done to reflect differences in its latitude dependence when the value of $b$ increases from 0.46 to 1.16 in Eq. (9). Therefore, the right panel of Fig. (1) provides the context to study the effects associated with the down-scaling of particle drifts as a function of heliolatitude (polar angles).

In this work, a 3D drift-model, as described below, is applied to study the effects on computed galactic proton and anti-proton intensities in the heliosphere for different drift reduction scenarios, as illustrated in Fig. (1). Of particular interest is how the spatial dependence of $\omega \tau$ and $f_{s}$ reduces differences in the level of modulation between the two drift cycles at the Earth from solar minimum to moderate maximum modulation conditions. Furthermore, it is also shown how the assumed drift reduction alters the anti-proton to proton ratio at Earth for the periods mentioned above.
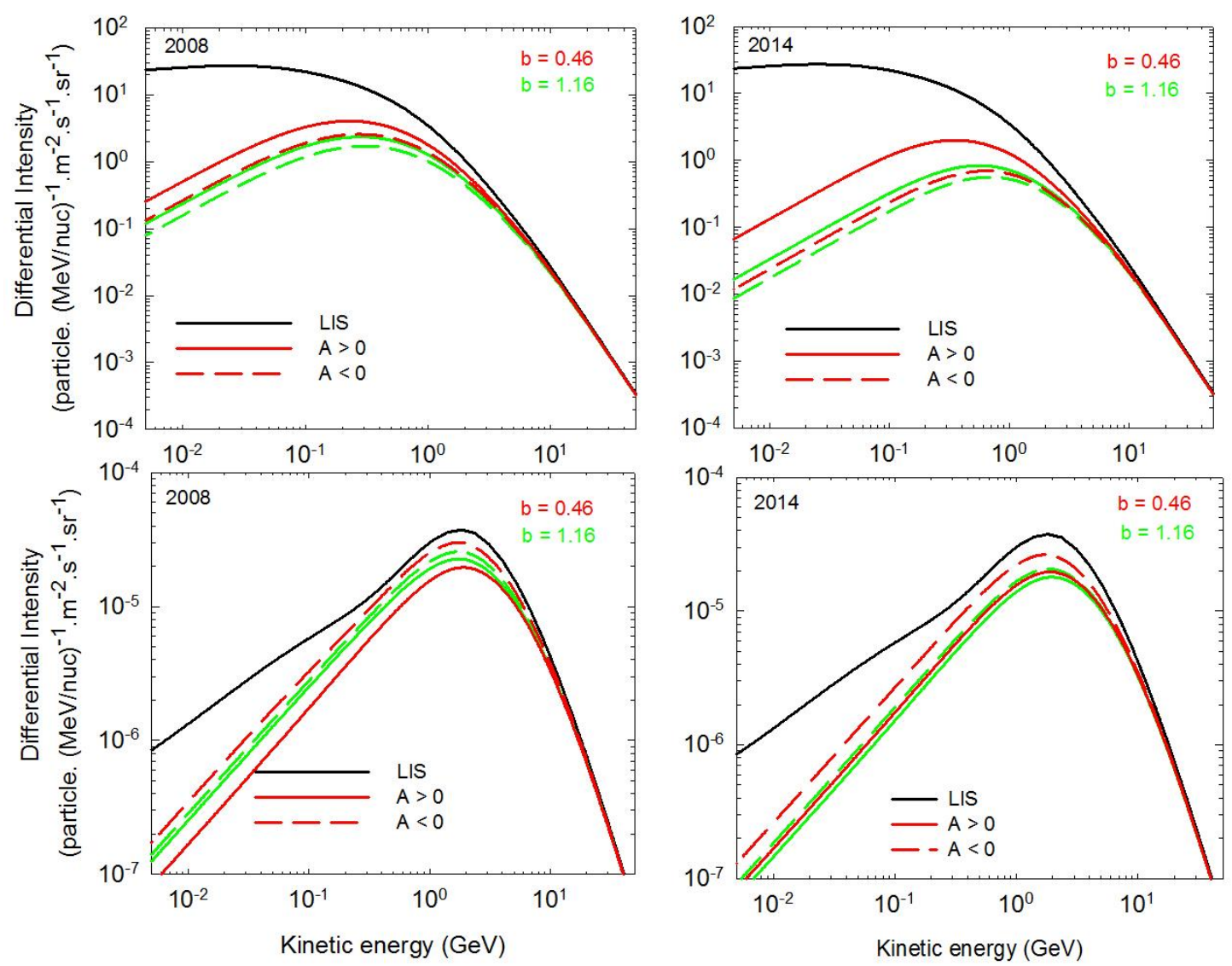

Fig.2: Top row: Computed spectra for GCR protons as a function of kinetic energy for the $A>0$ polarity cycle (solid lines) and the $A<0$ polarity cycle (dashed lines) in the equatorial plane at the Earth during solar minimum (left panel denotes 2008) and moderate maximum conditions (right panel denotes 2014). Two different spectra are shown which correspond to the two scenarios of $\lambda_{\mathrm{A}}$ as shown in Fig. (1); red lines represent solutions obtained with $b=0.46$ and green lines with $b=1.16$ in Eq. (9). The bottom row is similar to the top row but anti-proton spectra are shown with respect to the corresponding LIS which is significantly different from the proton LIS in the top panels. 


\section{$3 \quad$ Modulation model and the results}

The numerical model used in this study is based on solving the transport equation derived by [15]. The details and essentials of this numerical model together with the elements of the diffusion tensor have been published by [12] and [13]. The only parameter that is changed is the drift coefficient $K_{T}$, given by Eq. (6).

In Fig. (2) the modulation of protons (top row) and anti-protons (bottom row) is shown as spectra in the equatorial plane at $1 \mathrm{AU}$ for the $A>0$ polarity cycle (solid lines) and $A<0$ polarity cycle (dashed lines). The two different spectra shown in each panel correspond to two scenarios of $\lambda_{\mathrm{A}}$ as shown in Fig. (1), caused by $\omega \tau$ and $f_{s}$ in Eqs. (6) and (7). Here, the effects of $\omega \tau$ and $f_{s}$ are illustrated with emphasis on the resulting differences in the level of modulation between the two drift cycles. Comparing the four panels illustrate that increasing the value of $b$ in Eq. (9) from 0.46 to 1.16 reduces differences in modulation between the two drift cycles. Furthermore, this figure indicates that increasing the value of $b$ while simultaneously decreasing the value of $a$ in Eq. (9) seems plausible for modelling GCRs towards increasing solar activity.

Fig. (3) shows the computed anti-proton (pbar) to proton (p) ratios as a function of kinetic energy for $A<0$ polarity cycles (solid lines) and the $A>0$ polarity cycle (dashed lines) corresponding to spectra in Fig. (2). It is noted in both panels that the effects of varying the spatial dependence of $\omega \tau$ on the drift scale in the subsequent anti-proton to proton ratios are more prominent in the $A>0$ cycle.
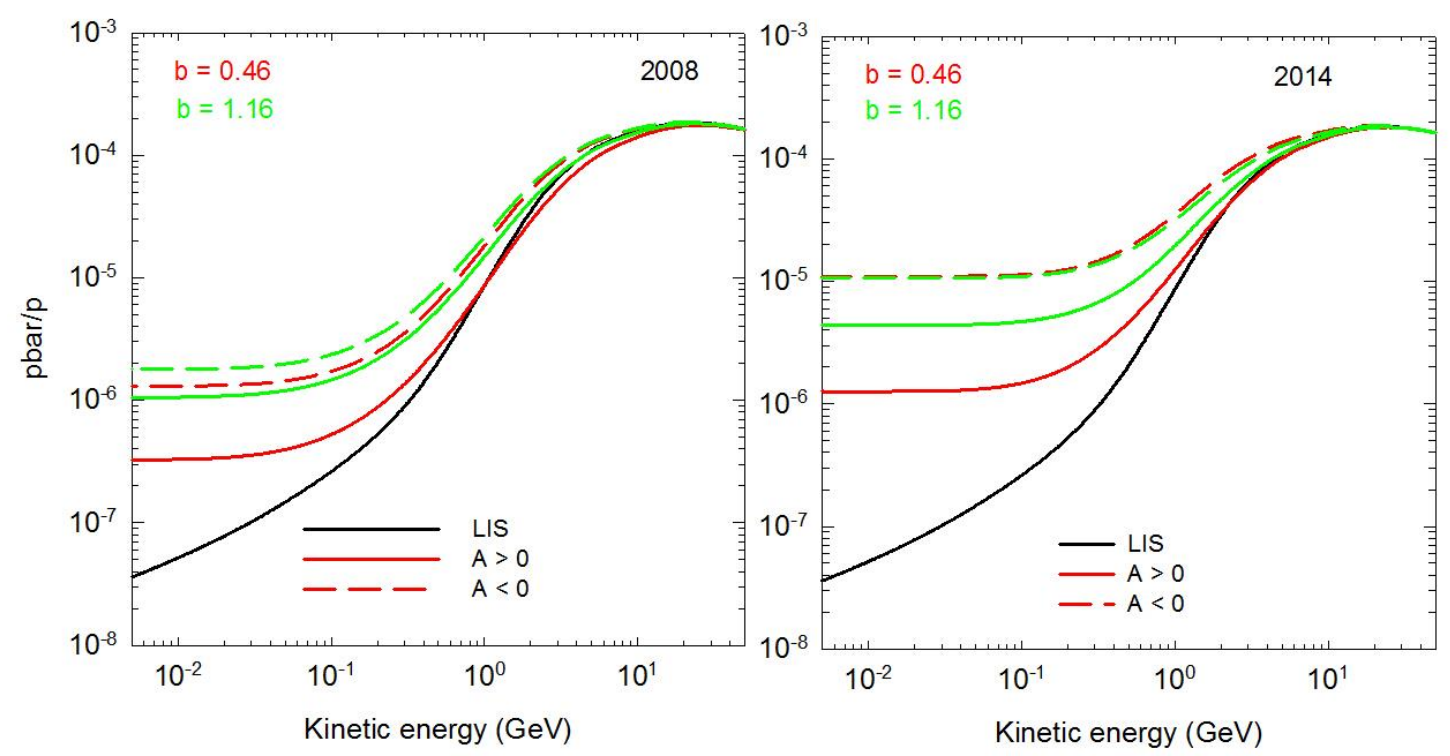

Fig.3: Computed anti-proton (pbar) to proton (p) ratios as a function of kinetic energy for the $A>0$ (solid lines) and $A<0$ polarity cycles (dashed lines) at the Earth (in the equatorial plane at $1 \mathrm{AU}$ ) during solar minimum modulation conditions (left panel; denotes 2008) and moderate maximum conditions (right panel; denotes 2014). Ratios are shown with respect to the corresponding ratio of the proton LIS and antiproton LIS. Two different ratios are shown corresponding to two scenarios of $\lambda_{\mathrm{A}}$ as shown in Fig. (1); red lines represent solutions obtained with $b=0.46$ and green lines with $b=1.16$ in Eq. (9).

\section{$4 \quad$ Summary and conclusion}

The 3D numerical drift model as described by [12] was used to study the effects of two different scenarios of $\omega \tau$, as given in Eq. (9), on the drift coefficient represented as a drift scale $\lambda_{\mathrm{A}}$ in Fig. (1), as applicable to the modulation of protons and anti-protons in the heliosphere. The two studied scenarios of $\varrho \tau$ are based on the work of [9]. The effects were illustrated for the two solar magnetic field polarities during solar minimum and moderate maximum modulation conditions. Of particular interest is how the spatial dependence of $\omega \tau$ and $f_{s}$ reduces differences in the level of modulation between the two drift cycles at the Earth; and how the anti-proton to 
proton ratio is altered from solar minimum to moderate maximum modulation conditions.

It is found that the assumed spatial dependence of $\omega \tau$ reduces differences in modulation between the two drift cycles, more so when the value of $b$ in Eq. (9) is increased. Therefore, Eq. (6) provides a simple and tractable way to study the effects of diffusive scattering on the drift coefficient over a solar cycle by adjusting the values of $a$ and $b$. Furthermore, it is found that the ratio of anti-protons to protons is less sensitive to the assumed spatial dependence of $ø \tau$ in the $A$ $<0$ cycle, especially during moderate maximum modulation conditions.

\section{Acknowledgements}

MDN thanks the SA National Research Foundation (NRF) for partial financial support under Joint Science and Technology Research Collaboration (Grant no: 118915).

\section{References}

[1] O. Adriani, G.C. Barbarino, G.A. Bazilevskaya, Astrophys. J.765, 91, (2013).

[2] N. Tomassetti, M. Orcinha, F. Barao, B. Bertucci, Astrophys. J. 849, 32, (2017).

[3] J. Feng and H. Zhang, Astrophys. J. 858, 116, (2018).

[4] M. Korsmeier and A. Cuoco, Phys. Rev. D. 94, 123019, (2016)

[5] M.S.Potgieter, Living Rev. Sol. Phys. 10, 3, (2013)

[6] U.W. Langner and M.S. Potgieter, J. Geophys. Res. 109, 2003

[7] N.E. Engelbrecht and R.A. Burger, Astrophys. J. 772, (2013).

[8] R.A. Burger and D.J. Visser, Astrophys. J. 725, 1366, (2010).

[9] R.C. Tautz and A. Shalchi, Astrophys. J. 125, 744-751, (2012).

[10] M.D. Ngobeni and M.S. Potgieter, Adv. Space Res. 53, 1634, (2014).

[11] N.E. Engelbrecht and R.A. Burger, Adv. Space Res, 55, 390, (2015).

[12] M.S. Potgieter and E.E. Vos, A\&A. 601, A23, (2017).

[13] D. Bisschoff , M.S. Potgieter, O.P.M. Aslam, Astrophys. J. 878: 59, (2019).

[14] J.W. Bieber and W.H. Matthaeus, Astrophys. J. 485, 655, (1997).

[15] E.N. Parker, Planet. Space Sci. 13, 9, (1965).

[16] O.P.M Aslam, M.S. Potgieter, D. Bisschoff. This conference, (2019).

[17] A. Cuoco, J. Heisig, L. Klamt, M. Korsmeier, M. Krämer, Phys. Rev. D. 99, 103014, (2019).

[18] I. Cholis, T. Linden, D. Hooper, Phys. Rev. D. 99, 103026, (2019).

[19] W.R. Webber and M.S. Potgieter, Astrophys. J. 344: 779 (1989). 